\title{
Four Decades of Studying Global Linear Instability: Progress and Challenges
}

\author{
F. Gómez, ${ }^{*}$ S. Le Clainche, ${ }^{\dagger}$ P. Paredes, ${ }^{\dagger}$ M. Hermanns, ${ }^{\star}$ and V. Theofilis ${ }^{\S}$ \\ Universidad Politécnica de Madrid, 28040 Madrid, Spain
}

\begin{abstract}
Global linear instability theory is concerned with the temporal or spatial development of small-amplitude perturbations superposed upon laminar steady or time-periodic three-dimensional flows, which are inhomogeneous in two (and periodic in one) or all three spatial directions. After a brief exposition of the theory, some recent advances are reported. First, results are presented on the implementation of a Jacobian-free Newton-Krylov time-stepping method into a standard finite-volume aerodynamic code to obtain global linear instability results in flows of industrial interest. Second, connections are sought between established and more-modern approaches for structure identification in flows, such as proper orthogonal decomposition and Koopman modes analysis (dynamic mode decomposition), and the possibility to connect solutions of the eigenvalue problem obtained by matrix formation or time-stepping with those delivered by dynamic mode decomposition, residual algorithm, and proper orthogonal decomposition analysis is highlighted in the laminar regime; turbulent and three-dimensional flows are identified as open areas for future research. Finally, a new stable very-high-order finite-difference method is implemented for the spatial discretization of the operators describing the spatial biglobal eigenvalue problem, parabolized stability equation three-dimensional analysis, and the triglobal eigenvalue problem; it is shown that, combined with sparse matrix treatment, all these problems may now be solved on standard desktop computers.
\end{abstract}

\section{Nomenclature}

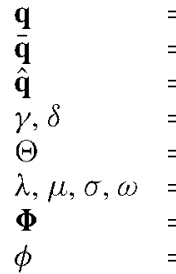

$=$

$=$

$=$

= Floquet multiplier, Floquet exponent

$=$ phase function

eigenvalues

propagator operator

$=$ empirical eigenfunction

Superscripts

$$
\begin{array}{ll}
\bar{q} & =\text { basic flow component } \\
\hat{q} & =\text { perturbation component } \\
* & =\text { adjoint operator }
\end{array}
$$

\section{Introduction}

$\mathbf{T}$ HERE is good reason for optimism regarding the future of global linear instability and a certain degree of euphoria surrounding its recent success $[1,2]$, three decades after the first solutions of multidimensional eigenvalue problems appeared in the literature $[3,4]$. In either its modal or nonmodal flavor, biglobal linear instability analysis, pertinent to flows developing in geometries with two inhomogeneous and one homogeneous spatial directions, are now routinely performed, while the first triglobal analyses, appropriate for fully three-dimensional flows, have also appeared in the literature [5-9]. Well-developed theoretical approaches for the description of linear systems, which in the context of classic linear stability theory (LST) had been endorsed with the infamous simplifying assumption of a 'parallel flow' to arrive at theoretically/ numerically tractable problems, can now be applied to more-realistic flow situations in which multiple inhomogeneous spatial directions exist. In the vast majority of real-world applications, the assumptions of parallel or quasi-parallel flow are not applicable; the scope of global linear theory in such flows keeps widening, in tune with the maturing of solution algorithms and the wider availability of computational infrastructure, both of which have made accessible to the wider scientific community the solution of problems previously reserved only for those groups with access to large-scale supercomputing facilities. The role of global linear analysis as a natural extension of the classic LST $[10,11]$ has always been clear, however, by contrast to the last two decades of the 20th century, during which activity in global instability analysis was rather scarce, the number of published work in this area is growing exponentially in the last decade.

This paper is not intended as an overview of the current state of the art in global linear instability research, nor is it meant as an in-depth discussion of any particular facets. The main objective of this paper is an attempt to contain the euphoria surrounding the potential of global linear instability theory, differentiate between what is currently feasible and what would be desirable to obtain from the analysis, and point, from a very subjective point of view, to some promising future research directions. In writing this paper, the authors wish to contribute to avoiding repetition of the disappointment that followed much-advertised past breakthroughs in research methodologies in fluid mechanics, such as quantitative description of turbulence via coherent structures, large-eddy simulation, and even direct numerical simulation, all of which were supposed to "solve the turbulence problem within the next decade" (this phrase will intentionally not be attributed to any one researcher, although wishful thinking to this tune still echoes in the ears of those who attended scientific meetings in the 1980s and 1990s).

The departure point of this paper is some thoughts exposed in Sec. II, where a list of open issues is presented that need to be addressed in the context of global linear instability analysis, thus providing a clear dichotomy between feasible and desirable information expected from the analysis. Section III presents a summary of current theoretical concepts underlying global linear instability analysis, while in Sec. IV answers to some of the issues raised, as 
recently provided by the authors, are presented. Concluding remarks close this paper in Sec. V.

\section{Feasible Versus Desirable Information Delivered by Global Linear Instability Analysis}

This section presents some thoughts on information that may be delivered by global linear instability analysis. Owing to the rapid expansion of this area, especially in the last decade, the real danger (or temptation) exists to unintentionally (or intentionally) misinterpret the scope of the theory. The following discussion is a contribution to ensuring elimination of confusion between the answers to two sets of questions, which differentiate between what is currently feasible against what is desirable to be obtained, although the authors are fully conscious (and hopeful) that progress toward obtaining the desirable information is continuous. In the first category, feasible information, one first identifies the following:

1) What is the information that can be obtained, given the current state of the art of the theory?

2) How does one presently obtain this information?

3) What can one do with global linear instability results today?

Then a list of desirable information is formulated:

1) What is the information that one would like to have access to?

2) How would one like to obtain it?

3) What would one wish to do with the aid of global linear instability analysis results?

Taking the first questions of the two lists together, on the one hand there exists the demonstrated ability to obtain biglobal eigenvalue problem (EVP) solutions and perform biglobal transient growth studies via either (direct/adjoint) initial value problem (IVP) or singular-value decomposition solutions, all of laminar steady or time-periodic two-dimensional flows, while a handful of triglobal EVP analyses also exist in the literature. On the other hand, it is clear that the instability of (laminar) flows with three inhomogeneous spatial directions is by and large unexplored from a global theory point of view. Order-of-magnitude improvements of the flexibility and the efficiency of the numerical methods used in this context is needed to achieve this target.

Related to the second dichotomy between feasible and desirable means to obtain global linear instability analysis results, the existence of a wide arsenal of well-validated algorithms to perform such analysis is known, mainly of academic (high-order accurate, high theoretical sophistication) flavor, and in addition hardware to support the related basic research is also available (supplemental material 3 in [1] discusses this point extensively). What would be desirable in this context would be to integrate ideas of global linear analysis into everyday engineering practice, using tools available to industry, e.g., large-scale aerodynamic calculation codes, provided of course that the global instability analysis community is capable of convincing industry of the utility of the theory, on the basis of experimentally cross-verified global instability analysis (and control) results. A clear stumbling block at present is turbulence modeling (in the context of instability analysis), which is known to work on certain classes of separated flows analyzed via local instability theory [12] and has also been demonstrated in the recent global analyses of Crouch et al. $[13,14]$. Nevertheless, much more needs to be done, e.g., along the lines of the recent work of Kitsios et al. [15].

Finally, it is important to mention what is perceived to be one of the major utilities of global instability analysis, namely its contribution to reduced-order modeling and flow control. Although the role of the theory in controlling linear instability of low-Reynolds-number steady or time-periodic two-dimensional laminar flows is welldocumented in the literature [16], the desired target is control of three-dimensional (three inhomogeneous spatial directions) turbulent flows; again, this is, in the authors' view, an area where advances are urgently needed.

The following discussion goes into some depth in each of the previous points; the index built up in the previous section aids structuring the exposition. Representative examples of the current state of the art introduced in Sec. III serve as cases in the points made. It should also be stressed that, in the interest of space, the scope of the following discussion is kept within the realm of linear global instability analysis; the reader interested in the ongoing vigorous developments in the (related, in case of multiple inhomogeneous spatial directions) theoretical approaches for nonlinear flow analysis [17-19], reduced-order modeling for complex flows [16,20-22], and flow control $[23,24]$ is referred to the original works and citations therein as well as to a recent monograph on a reduced-order model (ROM) [25]. Owing to the rapidly expanding nature of the global linear instability field, the list of works selected to be discussed is certainly partial and probably biased toward the authors' own research interests; apologies are offered to researchers whose interesting work is not discussed herein.

\section{Theoretical Considerations}

A short reminder of theoretical concepts underlying global linear instability analysis is necessary to set the scene for the discussion that follows. Our concern is with the development in time and space of small-amplitude perturbations superposed upon a given laminar flow. This can be described exactly by the linearized Navier-Stokes, continuity, and energy equations, without the need to invoke the parallel (or weakly nonparallel) flow assumption; the flow analyzed with respect to its global stability may be any laminar two- or threedimensional solution of the equations of motion, as well as flows with strong dependence on two inhomogeneous and weakly varying along the third spatial direction. The respective theoretical concepts are referred to as biglobal, triglobal, and parabolized stability equation three-dimensional (PSE-3D) analyses. Table 1 classifies and refines the different kinds of linear stability theory, demarcating the boundaries between local analysis based on variants of the Orr-Sommerfeld equation (OSE), nonlocal analysis based on the standard PSEs [26-28], which represent a generalization of the parallel-flow LST for flows with a mild variation on the streamwise direction, and the three aforementioned versions of global linear theory; symbols appearing will be defined shortly.

Linearization of the equations of motion may be performed around steady or unsteady laminar basic flows, $\bar{q}=(\bar{\rho}, \bar{u}, \bar{v}, \bar{w}, \bar{T})^{T}$. This is to be contrasted against some of the current global instability literature, which concerns time-averaged turbulent flows, which will not be dealt with in the present paper, although some comments on the applicability of the theory, supported by the still-scarce evidence in the literature, will be made in what follows.

Basic flows in complex geometries are typically provided by twoor three-dimensional direct numerical simulations, potentially exploiting spatial invariance. Steady laminar flows exist only at low Reynolds numbers, but numerical procedures exist for the recovery of basic flows also at conditions where linear global instability would be expected, e.g., based on mirroring the solution computed under the imposition of symmetries, continuation [29], or selective frequency damping [30]. In using the term small-amplitude perturbations, the decomposition

Table 1 Classification of global linear theory approaches

\begin{tabular}{lcccc}
\hline \hline & Denomination & Basic state & Assumptions & Phase function $\Theta$ \\
\hline \multirow{2}{*}{ Global } & Triglobal & $-\bar{c}$ & $\bar{q}\left(x_{1}, x_{2}, x_{3}\right)$ & $\omega t$ \\
& PSE-3D & $\partial_{1} \bar{q} \ll \partial_{2} \bar{q}, \partial_{3} \bar{q}$ & $\bar{q}\left(x_{1}^{*}, x_{2}, x_{3}\right)$ & $\int \alpha\left(x_{1}^{*}\right) \mathrm{d} x_{1}^{*}-\omega t$ \\
Nonlocal & Biglobal & $\partial_{1} \bar{q}=0$ & $\bar{q}\left(x_{2}, x_{3}\right)$ & $\alpha x_{1}-\omega t$ \\
Local & PSE & $\partial_{1} \bar{q} \ll \partial_{2} \bar{q} ; \partial_{3} \bar{q}=0$ & $\bar{q}\left(x_{1}^{*}, x_{2}\right)$ & $\int \alpha\left(x_{1}^{*}\right) \mathrm{d} x_{1}^{*}+\beta x_{3}-\omega t$ \\
\hline \hline
\end{tabular}




$$
\mathbf{q}=\overline{\mathbf{q}}+\epsilon \tilde{\mathbf{q}}, \quad \epsilon \ll 1
$$

is assumed, and solutions to the initial-value problem

$$
\mathcal{B}(R e, M a, \overline{\mathbf{q}}) \frac{\mathrm{d} \tilde{\mathbf{q}}}{\mathrm{d} t}=\mathcal{A}(R e, M a, \overline{\mathbf{q}}) \tilde{\mathbf{q}}
$$

are sought. Specific comments on the dependence of these quantities on the spatial coordinates $\mathbf{x}$ and time $t$ will be made in what follows. The operators $\mathcal{A}$ and $\mathcal{B}$ are associated with the spatial discretization of the linearized equations of motion and comprise the basic state $\overline{\mathbf{q}}(\mathbf{x}, t)$ and its spatial derivatives. In case of steady basic flows, the separability between time and space coordinates in Eq. (2) permits introducing a Fourier decomposition in time:

$$
\tilde{\mathbf{q}}=\hat{\mathbf{q}} e^{i \Theta}
$$

with $\Theta$ as a phase function, leading to the generalized matrix eigenvalue problem:

$$
\mathbf{A} \hat{q}=\omega \mathbf{B} \hat{q}
$$

Here, matrices $\mathbf{A}$ and $\mathbf{B}$ discretize the operators $\mathcal{A}$ and $\mathcal{B}$, respectively, incorporating the boundary conditions; $\hat{\mathbf{q}}(\mathbf{x} ; t)=(\hat{\rho}, \hat{u}, \hat{v}, \hat{w}$, $\hat{T})^{T}$ is the vector comprising the amplitude functions of linear density, velocity component, and temperature or pressure perturbations. The eigenvalue problem adjoint to Eq. (4) may also be derived, after suitable definition of an inner product, typically associated with perturbation energy in incompressible [11] and compressible [31] flow, and enforcement of the bilinear concomitant to zero [32]. Both the direct EVP [Eq. (2)] and its associated adjoint describe a modal global linear instability scenario, applicable to the dynamic behavior of the linearized equations of motion at the asymptotic limit $t \rightarrow \infty$.

\section{A. Linear Global Modes}

In what follows, solutions of Eq. (4) are referred to as global modes and are, by definition, linear. Physical interpretation of the quantities appearing in Eq. (4) depends on whether a (temporal or spatial) biglobal or triglobal context of analysis is followed; initialization of PSE-3D at the start of the marching location requires solution of the spatial biglobal EVP.

The key assumption underlying both temporal and spatial biglobal instability analysis is that the basic state is spatially invariant along one spatial direction, say $x_{1}$. In temporal biglobal analysis the physical interpretation of quantities appearing in Eq. (4) is as follows: the wave number $\alpha$, appearing in Table 1, is a real parameter and is related with a periodicity length $L_{x_{1}}$ along the homogeneous direction $x_{1}$ through $\alpha=2 \pi / L_{x_{1}}$. In a manner analogous with classic LST, the eigenvalue $\omega$ is complex, composed of a real part $\Re\{\omega\}$, which is related with the global mode frequency, and an imaginary part $\Im\{\omega\}$, which is the temporal amplification $(\Im\{\omega\}<0)$ or damping $(\Im\{\omega\}>0)$ rate of the global mode. In spatial biglobal analysis, $\omega$ is a real frequency parameter, and the linear EVP [Eq. (4)] is rewritten as an EVP for the determination of the eigenvalue $\alpha$. The obtained result $\alpha=\Re\{\alpha\}+i \Im\{\alpha\}$ may be used to identify a periodicity length $L_{x_{1}}=2 \pi / \Re\{\alpha\}$ along the homogeneous spatial direction $x_{1}$, while $-\Im\{\alpha\}$ is the spatial amplification rate.

Both the spatial discretization and the numerical solution of the global EVP itself are well-documented in several sources (e.g., [1]) and will not be dwelled upon here. Attention will only be brought upon the fact that temporal biglobal theory leads to a linear EVP, while the spatial analog of this theory requires solution of a quadratic eigenvalue problem. Nonlinear EVPs may be converted to linear ones by the companion matrix approach $[33,34]$, which, in the problem at hand, multiplies the memory requirements of a temporal biglobal analysis by a factor 4 , if a dense storage scheme is used, while the CPU time necessary for its solution is larger by a factor 8 compared with the temporal biglobal EVP, if a direct matrix inversion approach is employed. In this sense, not only is research into alternative direct solution methods urgently needed but also matrix-free formation methods, which contribute to reducing the computational resources required will be discussed in Sec. IV. Finally, the situation regarding boundary conditions to close the PDE-based EVPs is clear as far as solid walls are concerned but not so for open flows. To provide insight to this issue, Rodríguez et al. [35] undertook an effort to quantify the effect of upstream and downstream boundary conditions in simple two-dimensional problems in which progress can also be made by analytical tools.

Turning to the PSE-3D, $\omega$ is a real parameter and, in a manner analogous with classic LST and PSE applied to boundary-layer flows, initialization data may be provided by solution of the spatial biglobal EVP. The satisfaction of the normalization condition delivers the results for the wave number and amplification rate, respectively to be compared with $\Re\{\alpha\}$ and $\Im\{\alpha\}$ delivered by the spatial biglobal analysis. A PSE-3D methodology for the instability analysis of realistic isolated vortices and systems of vortical flows is discussed in detail by Paredes et al. [36].

Triglobal modal linear instability analysis may also be performed by numerical solution of Eq. (4), whereby the amplitude functions are three-dimensional functions of three inhomogeneous spatial coordinates. The analysis is always temporal, the only parameter being the flow Reynolds number, while the physical interpretation of the real and imaginary parts of the eigenvalue $\omega$ is the same as in classic LST and temporal biglobal instability analysis; triglobal EVP solutions have appeared in the literature in the last decade [5-9]; a contribution in this direction may be found herein using an alternative matrix formation method.

Independent of the numerical integrity of the global eigenvalue problem results, the ultimate criterion regarding their relevance to flow analysis is comparison with physical reality. If modal global linear instability results are coherent with those recovered from experiment and direct numerical simulation (DNS), under conditions of natural (not controlled) transition initialization, it may be concluded that modal global linear theory is sufficient to describe amplification of small-amplitude disturbances; otherwise, nonmodal global theory must be used.

\section{B. Relation Between Numerical Residuals and Global Modes}

For the sake of completeness of the presentation that follows, which focuses in extraction of global mode results from transient simulation data or experiment, the residual algorithm (RA) will be summarized next.

It is possible to extract information about the damping rate and amplitude functions of decaying linear global modes from the transient data of time-accurate integration of the equations of motion toward a steady state. In particular, if the time-accurate integration is close to convergence, then the decomposition [Eq. (1)] is valid, being straightforward to obtain

$$
\sigma=\ln \left[\frac{\mathbf{q}^{\mathbf{t}}}{\mathbf{q}^{\mathbf{t}-\Delta \mathbf{t}}}\right] / \Delta t \approx \frac{\mathrm{d} \ln \left[\mathbf{q}^{\mathbf{t}}\right]}{\mathrm{d} t}
$$

where $\mathbf{q}^{\mathbf{t}}=|\mathbf{q}(\mathbf{x}, \mathbf{y}, \mathbf{z}, \mathbf{t})-\overline{\mathbf{q}}|$ is obtained from the DNS transient data, and $\sigma$ is identified as the damping ratio corresponding to the global modes. In addition, it is possible to extract amplitude functions and frequency of the global modes or recover the converged solution from transient data following the same idea; further details may be found in $[1,37]$.

\section{Strobes (Snapshots), Chronoi (Times), and Topoi (Spaces)}

The 1980s and 1990s of last century witnessed exciting developments in the field of deterministic description of coherent structures of turbulent flows. Application of the Karhunen-Loève (K-L) theory, also known in different fields of computational mechanics as proper orthogonal decomposition (POD) [38] and principal component analysis, permits reduction of large space-time collections of numerical or experimental laminar or turbulent flowfields by solution of an eigenvalue problem for the determination of a small number of eigenmodes known as $\mathrm{K}-\mathrm{L}$, POD, or empirical eigenmodes, the latter denomination alluding to the fact that knowledge of the underlying dynamical operator is not necessary for the construction 
of the empirical eigensystem. Concretely, given a sequence of instantaneous data $\mathbf{u}_{i} \equiv \mathbf{u}\left(\mathbf{x}, t_{i}\right)$, one forms the cross-correlation tensor

$$
\mathcal{R}\left(\mathbf{x}, \mathbf{x}^{\prime}\right)=\left\langle\mathbf{u}(\mathbf{x}) \mathbf{v}\left(\mathbf{x}^{\prime}\right)\right\rangle
$$

or autocorrelation tensor, when $u=v$, with $\mathcal{R}$ being a compact selfadjoint operator [38], and solves the EVP

$$
\int_{\partial \Omega} \mathcal{R}\left(\mathbf{x}, \mathbf{x}^{\prime}\right) \phi\left(\mathbf{x}^{\prime}\right) \mathrm{d} \mathbf{x}^{\prime}=\lambda \boldsymbol{\phi}(\mathbf{x})
$$

to determine the empirical eigenmodes $\phi(\mathbf{x})$. This description has (at least) two major attractive features. First, unlike the global eigenmodes, solutions of Eq. (4) or the Koopman modes, obtained through solution of Eq. (15), the empirical eigenfunctions are orthogonal, permitting expansion of (nominally) arbitrary fields onto the latter eigensystem. Second, most of the system energy is captured by a relatively small number of empirical eigenfunctions, which forms the basis for subsequent ROM efforts for the efficient description of the original system [25].

Sirovich [39] introduced the method of snapshots or method of strobes to simplify calculation of the correlation-tensor-based eigenvalue problem [Eq. (7)] in case of short time samples and highly resolved data. This is of particular interest to analyzing transitional data, because long time integration is unnecessary during the linear regime, and high spatial resolution is desirable. Of particular interest in the present context is that the simplifying assumptions of spatial homogeneity, invoked in the original works to make the problem of calculation of empirical eigenfunctions tractable from a numerical point of view, are not necessary if the snapshots method [39-41] is used.

Sirovich [39] proceeded to expand the empirical eigenfunctions as linear combinations of the instantaneous flowfields,

$$
\boldsymbol{\phi}=\sum_{j=1}^{M} \alpha_{j} \mathbf{u}
$$

and arrive at the eigenvalue problem

$$
\mathrm{C} \phi=\lambda \phi
$$

This is equivalent to Eq. (7) in terms of obtaining identical eigenvalues, circumvents tedious and impractical for large 3D problems calculation of the kernel [Eq. (6)], and is thus substantially more efficient than the original eigenvalue problem because the size of matrix $\mathbf{C}$ depends on the number of realizations (snapshots or strobes) $M$, used in its construction via

$$
C_{j k}=\frac{1}{M}\left(\mathbf{u}_{j}, \mathbf{v}_{k}\right)
$$

and not on the original problem dimension. As noted in [41], the empirical eigenfunctions $\phi$ are not themselves coherent structures but may rather be used as the appropriate basis for the decomposition of the coherent structures through decomposition of any flowfield into the empirical eigenfunctions

$$
\mathbf{q}(\mathbf{x})=\sum_{j} a_{j} \boldsymbol{\phi}_{j}(x)
$$

Although this discussion is equally applicable to biglobal and triglobal linear instability, in the former context it would be possible to seek empirical eigenfunctions by exploiting translational invariance along the homogeneous direction and further decompose linear perturbations into harmonic functions along the homogeneous and an inhomogeneous two-dimensional part along the remaining two spatial directions. Rempfer and Fasel [42] argued against such a procedure, reasoning that it conceptually contradicts the idea of a compact coherent structure. Instead, they used the concept of spatiotemporal interchangeability and the bi-orthogonal expansion introduced by Aubry et al. [43] to decompose a given flowfield as

$$
\mathbf{q}\left(\mathbf{x}, t_{j}\right)=\sum_{i} \zeta_{i}\left(t_{j}\right) \sigma_{i}(\mathbf{x})
$$

where each time-dependent expansion coefficient $\zeta_{i}$ was termed 'chronos' (time), while the space-dependent coefficient $\sigma_{i}$ was called 'topos' (space).

\section{Koopman Modes and Dynamic Mode Decomposition}

The rather old concept of Koopman modes [44] has been recently introduced to the analysis of fluid flow structures by Rowley et al. [17] and Schmid [45], as a particular class of techniques for nonlinear systems analysis and reduction discussed in the influential work of Mezić [46]. The Koopman operator is a linear operator defined for any nonlinear dynamical system, thus allowing for the spectral analysis of nonlinear flows and flow coherent structures description. The Koopman modes reduce to linear global modes when the dynamical system is governed by the linearized Navier-Stokes operator, while in case of time-periodic flows the Koopman modes reduce to Fourier modes. For the sake of completeness in this section, the dynamic mode decomposition (DMD) algorithm presented by Schmid et al. [47] is briefly described. The existence of spatial and temporal decomposition concepts open different possibilities of structural analysis with the same tool; however, in this paper, attention is focused on temporal analysis.

Given a sequence of $N+1$ instantaneous data $\mathbf{v}_{i} \equiv \mathbf{v}\left(\mathbf{x}, t_{i}\right)$, two different snapshots matrices can be constructed: $V_{1}^{N}=\left\{v\left(x, t_{1}\right)\right.$, $\left.v\left(x, t_{2}\right), \ldots, v\left(x, t_{N+1}\right)\right\}$ (from the first snapshot to the $N$ th snapshot) and $V_{2}^{N+1}=\left\{v\left(x, t_{2}\right), v\left(x, t_{3}\right), \ldots, v\left(x, t_{N+1}\right)\right\}$ (from the second snapshot to the $(N+1)$ th snapshot). It is worth noting that, in a manner analogous to data analyzed by POD, snapshot data may arise either from simulation or experiments; in what follows, DNSobtained results will be employed to apply the algorithm and compare its results with those of global mode analysis based on numerical solution of Eq. (4).

Assuming a constant linear mapping $A$ over the entire snapshot sequence, which in general represents a nonlinear system and connects the flowfield $v_{i}$ with the subsequent flowfield $v_{i+1}$, i.e., $v_{i+1}=\mathbf{A} v_{i}$, it is possible to formulate the sequence of flowfields as a Krylov sequence:

$$
V_{2}^{N+1}=\mathbf{A} V_{1}^{N}
$$

Selecting a companion matrix that can be thought of as a projection of $A$ onto the snapshot basis $V_{1}^{N} \mathrm{Eq}$. (13) can be approximated by

$$
V_{2}^{N+1} \simeq V_{1}^{N} \mathbf{S}
$$

The idea behind DMD is to first construct $\mathbf{S}$ from the snapshot data and then describe the dynamical process defined by $A$ (and approximated by $S$ ) through numerical solution of the eigenvalue problem

$$
\mathbf{S} \mu=\lambda \mu
$$

where the dynamical modes $\Phi$ are the projection of the eigenvectors $\mu$ on the snapshot basis $V_{1}^{N}$,

$$
\boldsymbol{\Phi}=\sum_{j=1} \mu_{j}\left(V_{1}^{N}\right)_{j}
$$

and the eigenvalues follow the next transformation $\omega=\log (\lambda) / \Delta t$, where $\Delta t$ is the time interval between snapshots.

\section{E. Relation Between Numerical and Empirical Eigenmodes}

The main idea behind comparisons of global, POD, and Koopman modes is to investigate the relation between numerical and experimental results because, as opposed to global instability analysis, which requires a well-defined steady or time-periodic basic state, both POD and DMD analysis can be applied to either numerical or experimental (sparse) data. In addition, application of flow 
topological ideas to global instability analysis results has demonstrated that long-known flow structures, such as the stall cells preceding wing stall [48] and U-separation associated with adversepressure-gradient boundary layer flow on a flat-plate [49], can be uniquely and unequivocally defined as arising from linear amplification of global modes of the respective flows.

The principal motivation of the application of the K-L theory/ POD to fluid flow has been to provide a description of turbulence based on deterministic coherent structures, the latter reconstructed using the empirical eigensystem delivered by $\mathrm{K}-\mathrm{L} / \mathrm{POD}$ analysis [38]. By construction of the operator, this POD analysis identifies the most-energetic structures in the flow as POD modes.

Rowley et al. [50,51] extended the combined POD/LST analysis to two inhomogeneous spatial dimensions, studying DNS-obtained compressible flows over open cavity configurations. They demonstrated that a reduced-order model could be constructed, based on a relatively small (compared with that required for the DNS) number of POD modes satisfying Eq. (12). They also compared the spatial structure of the leading POD modes with that of the (then available) local, parallel LST and observed certain analogies in the two sets of data in the unstable shear-layer region at the open end of the cavity.

In the same direction, recent research by Sengupta et al. [52] in the analysis of the flow past a circular cylinder has linked the POD modes and the instability modes of this flow by means of nonlinear interactions satisfying the Landau-Stuart-Eckhaus equation and also found qualitative analogies between LST and POD mode results.

Merzari et al. [53] applied POD analysis to turbulent flows in geometries using the snapshots method containing a narrow gap, thus extending their earlier global linear instability analysis of the same flows in the laminar regime [54]. These authors provided evidence by comparison of such POD eigenfunctions on the one hand, and results of their earlier biglobal instability analysis [54] that the leading POD eigenfunctions of turbulent flow exhibit strong spatial analogies with the amplitude functions of the leading biglobal eigenmodes.

Finally, Oberleithner et al. [55] very recently presented direct comparison of empirical modes and linear stability eigenmodes of swirling-jet flow undergoing vortex breakdown. Besides the by now standard snapshot approach for the calculation of POD eigenmodes [39], these authors employed classic OSE- and PSE-based linear stability analysis and the triple-decomposition [56] concept to analyze the turbulent data extracted from their experiment. To the authors' knowledge and reservations from a theoretical point of view aside (regarding the reconstruction from nonlocal data of the global mode, as opposed to performing a PSE-3D or triglobal instability analysis), Fig. 18 of Oberleithner et al. [55] is the first attempt at comparison and the first conclusive demonstration of excellent agreement between the leading POD and the leading triglobal global flow eigenmode.

The results of Rowley [50,51], Sengupta [52], Merzari et al. [53], and Oberleithner et al. [55] in comparing empirical and global flow eigenmodes in two and three inhomogeneous spatial directions, respectively, demonstrate that such analysis may be feasible. It is certainly also desirable, in the sense that it paves the way to description of the flow by simpler models and, ultimately, to its control. Although speculative at this time, the same successes may both be attributable to the predominance of a single Fourier harmonic in the flow dynamics. It is worth examining how the situation may be different in (complex) flows in which additional frequencies are present, what the dependence of analogous comparisons between leading global and leading POD eigenmodes on the relative significance of the additional frequencies in the overall spectrum is, and ultimately where the boundary may be defined between phenomena, which can be attributed to linear dynamics, as opposed to flows in which consideration of nonlinearity is essential.

As far as DMD is concerned, this analysis was applied to a jet in crossflow [45], and the results were compared to the POD analysis and global modes. In particular, they found that the Koopman modes eigenfunction are similar to the POD modes, which describes the most energetic structures. However, the POD modes contains several frequencies while the Koopman modes only have one frequency by construction; therefore, they can separate the dynamics of the system more effectively, although the physical interpretation of that decomposition may not be clear. The most interesting results is that the Koopman frequency matches with the shedding frequencies obtained with the DNS. In addition, according to them, the global eigenmodes capture only the dynamics in a neighborhood of the unstable fixed point, while the Koopman modes correctly capture the behavior on the attractor. The present lack of sufficient information on all these $\mathcal{O}(1)$ issues, in either laminar or in the more interesting from an application point of view turbulent flows, is one of the key reasons to contain the euphoria [57]; future research should aim at resolving these issues.

\section{Some Recent Developments}

This section is used to present some recent work developed by the authors on a number of the fronts identified in the earlier discussion. First, attention is paid to the somewhat artificial dilemma of whether to perform global linear instability in a matrix-forming or a timestepping framework, which has plagued the community of global instability practitioners since its formation. Supplemental appendix 3 of [1] presents a rather comprehensive table showing that both approaches for the recovery of leading flow global eigenmodes have successfully been in use side by side, right from the early days of the analysis. Initially, this question could be paraphrased as "What type of computing infrastructure is available for the analysis?" with the broad answer being that authors with access to large-scale facilities could afford full-spectrum calculations, while all the rest had to conform with time-stepping methodologies. Subsequently, the question is addressed, regarding the degree to which the RA, DMD, and POD algorithms may be used to extract quantitative information from the same simulation data used in the time-stepping (TS) algorithm discussed in the next subsection; the square lid-driven cavity, the global linear instability of which is well-documented, is chosen as a test-flow for this exercise. Next, attention is turned to yet another controversial issue, that of whether to perform global linear instability analysis by forming the matrix or not. A new high-order accurate stable finite-differencing scheme [58] is employed to spatially discretize the linear operators pertinent to the PSE-3D [36] and the triglobal eigenvalue problem, and the efficiency gains offered compared with classical spectral collocation discretization are discussed.

\section{A. Time-Stepping with Low-Order Finite-Volume Methods}

Introduction of time-stepping ideas into standard aerodynamics codes, with the aim of broadening the scope of the analysis in transonic, supersonic, and hypersonic flow over or through complex geometries, is a nontrivial task, on account of the potentially distinct requirements of the robustness needed for aerodynamics codes to converge versus the accuracy needed for stability calculations. Although the majority of spatial discretization methods employed in computational fluid dynamics (CFD) have also been used for the solution of the global EVP in a matrix-formation context [1], the vast majority of time-stepping approaches to solve the same problem have been implemented in conjunction with incompressible flow and high-order (typically spectral-element) spatial discretization. In following the latter path, some of the most interesting flow phenomena associated with compressible flow have remained out of the scope of global instability analysis.

Although this situation is slowly changing in recent literature $[13,31,59]$, global instability analysis is yet to penetrate into the world of classic CFD schemes, the latter typically being based on variants of low-order finite-volume methods. Gómez et al. [60] recently presented a Jacobian-free Newton-Krylov-based timestepping algorithm of the class introduced into global instability analysis by Eriksson and Rizzi [61] and successfully employed to analyze biglobal $[62,63]$ and triglobal [5] EVP, combined with a standard finite-volume solver for compressible flow capable of addressing compressible flows over complex geometries. Validation results have been obtained by reference to the classic twodimensional square lid-driven cavity flow, one of which is shown in Fig. 1 at $R e=200, \beta=0.01$. $\mathrm{SC}$ is spectral collocation, $\mathrm{FV}$ is finite 


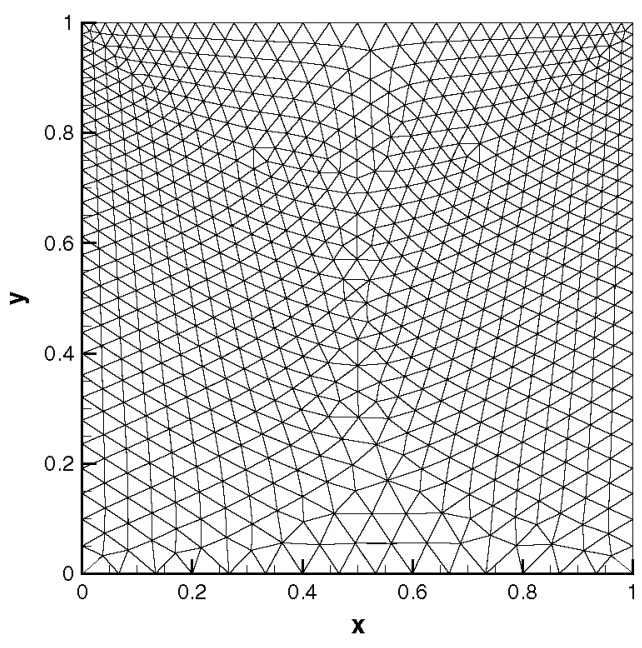

a) FV mesh

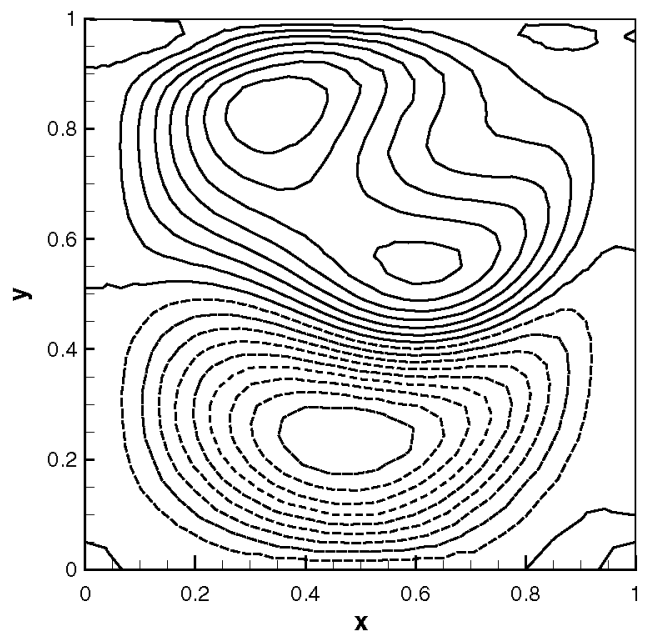

c) $\hat{\mathbf{n}}_{\mathrm{FV}}(\boldsymbol{x}, \boldsymbol{y})$

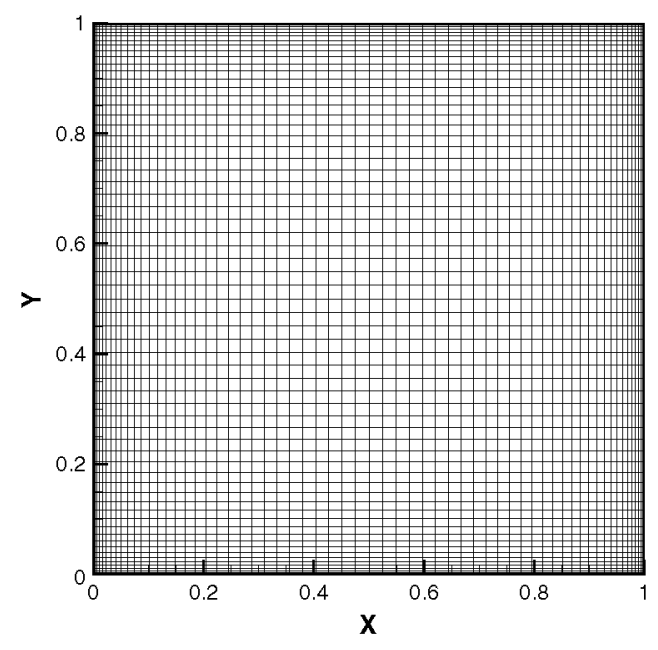

b) SC mesh

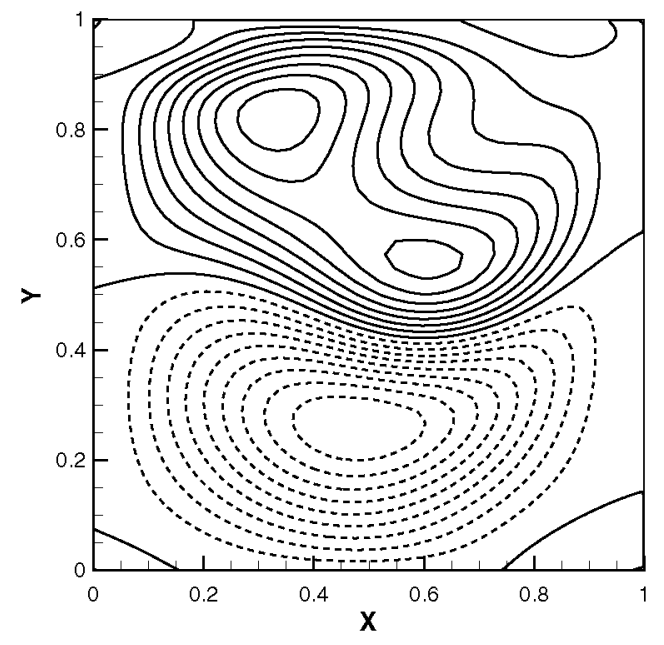

d) $\hat{\mathbf{u}}_{\mathrm{SC}}(\boldsymbol{x}, \boldsymbol{y})$

Fig. 1 Leading (damped) eigenmode obtained using DNS and a Jacobian-Free Newton Krylov (JFNK) time-stepping method [63,64] at Re $=200$.

volumes. Eigenvectors are normalized with $\hat{u}_{\max }$ and $\hat{v}_{\max }$. Dashed lines mean negatives values; 21 equidistant isolines from $\hat{u}=-1$ to $\hat{u}=1$ and from $\hat{v}=-1$ to $\hat{v}=1$; further details may be found in [60].

\section{B. Structures Identification}

As a nontrivial example of structures identification, linear global modes of the two-dimensional lid-driven cavity flow have been obtained by solution of the biglobal (BG) eigenvalue problem [Eq. (4)] on $64 \times 64$ spectral collocation points at subcritical conditions ( $R e=2000, \beta=0.0001$ ) as well as by performing DMD, TS, RA, and POD analyses of DNS-obtained solutions. This flow is known to be stable [65] and comprise both stationary and traveling decaying modes. The objective of this section is to compare the performance of the different approaches in describing these structures. For the DNS work, the flow is calculated with spectral collocation for the spatial discretization and the algorithm proposed by Spalart and Rogers in [66] for the temporal discretization. More details about the DNS can be found in [2]. The Chiba approach [62] has been followed in the TS analysis, with a random linear perturbation superimposed upon the base flow used as initial condition, and a total integration time $t=40$. In this case, a small Krylov subspace dimension $(m=6)$ is sufficient to accurately capture the first two flow eigenmodes. Regarding the frequency recovery, an aliasing-like problem occurs due to the relatively large integration time used in the DNS and the fact that the complex logarithm is a multivalued function. However, this problem is easily solved by analyzing the
Fourier transform of the DNS. To compute DMD/Koopman modes and POD, a sequence of 400 snapshots is taken, starting at $t=200$ with $\Delta t=2$, not sampling the transient time.

Figure 2 (upper) presents the leading eigenmode of lid-driven cavity flow at $R e=2000$ obtained by biglobal analysis and DMD, TS, RA, and POD analysis. In all cases, line-thickness agreement is seen in the respective results. The leading corresponding eigenvalue is compared in Table 2 for the first four methods. On the other hand, due to the existence of a strongly dominant linear instability (high kinetic energy level) over the flowfield pattern, the first POD topomode [see Eq. (12)] is able to capture its spatial structure. Biglobal analysis results are considered as converged reference. Relative error for the leading eigenvalue is $6.3 \cdot 10^{-5}$ for the DMD analysis, 1.2 . $10^{-3}$ for the TS analysis, and $3.4 \cdot 10^{-4}$ for the RA algorithm.

Figure 2 (lower) presents the second leading eigenmode of liddriven cavity flow at $R e=2000$ obtained by the first four methods (upper). Line thickness agreement is again recovered in the results of the first four methods. The second leading eigenvalue is also presented in Table 2. The relative error of the real part of the eigenvalue is $3.1 \cdot 10^{-3}$ for the DMD analysis, $3.9 \cdot 10^{-3}$ for the TS analysis, and $7.6 \cdot 10^{-3}$ for the RA algorithm, and the relative error for the imaginary part is $3.2 \cdot 10^{-2}$ for the DMD analysis, $5.2 \cdot 10^{-3}$ for the TS analysis, and $1.0 \cdot 10^{-1}$ for the RA algorithm. Eigenvectors are normalized with $\hat{u}_{\max }$ and $\hat{v}_{\max }$. Dashed lines mean negatives values; 21 equidistant isolines from $\hat{u}=-1$ to $\hat{u}=1$ and from $\hat{v}=-1$ to $\hat{v}=1$. Line-thickness agreement is obtained between the results of all algorithms employed. 


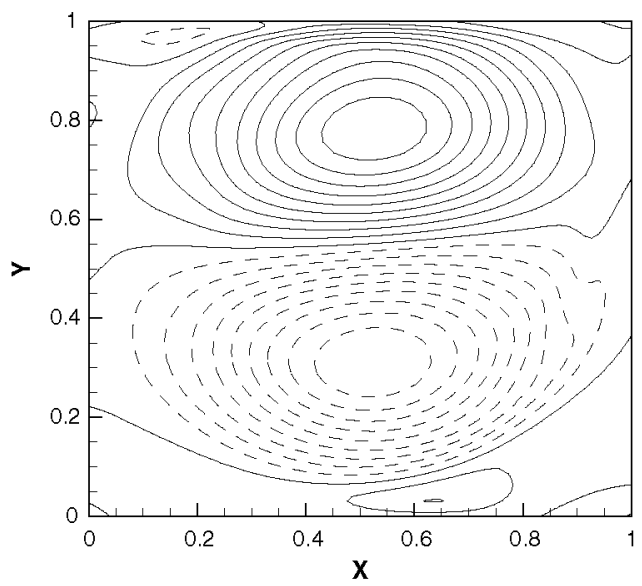

a) $\hat{\mathbf{u}}_{\mathrm{I}}(x, y)$

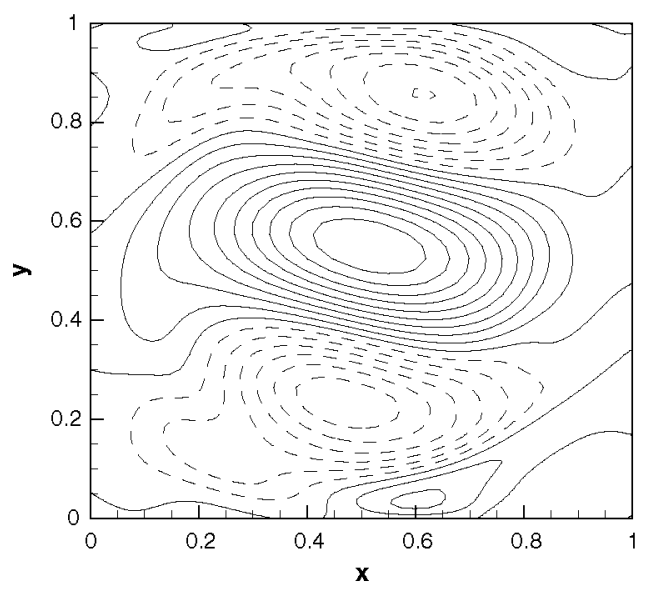

c) $\hat{\mathbf{u}}_{\mathrm{II}}(x, y)$

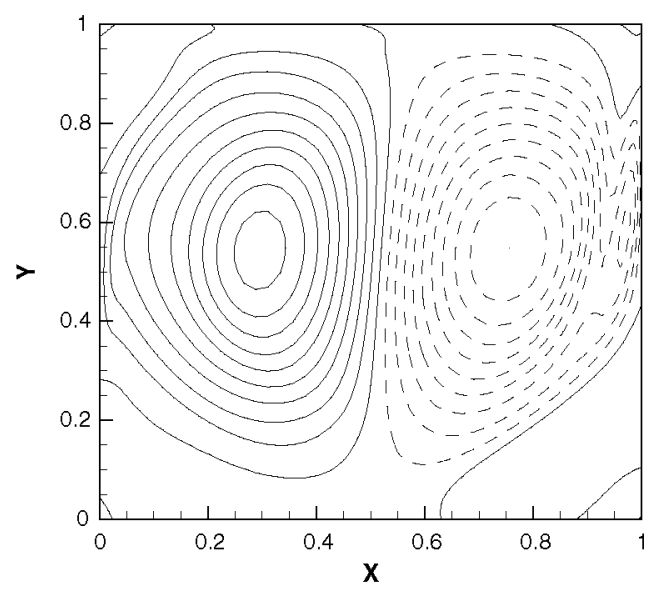

b) $\hat{\mathbf{v}}_{\mathrm{I}}(x, y)$

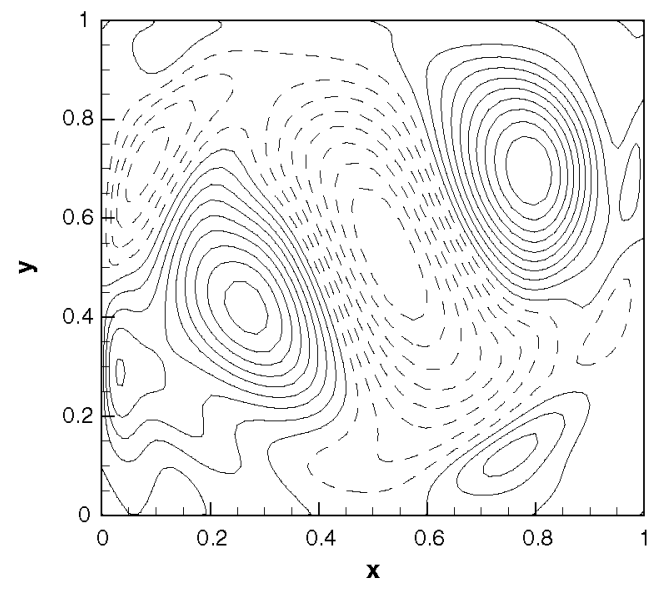

d) $\hat{\mathbf{v}}_{\mathrm{II}}(x, y)$

Fig. 2 Eigenmodes of the regularized lid-driven cavity (LDC) obtained using BG instability analysis, TS, Koopman analysis (DMD), and RA and first POD topo mode at $R e=2000$ a-b) first, c-d) second.

Figure 3 presents the damping ratio $\sigma$ obtained with the RA method. The damping rate presents a exponential decay with time and is a linear combination of the first, second, and third leading modes. Two traveling modes with frequency $\omega_{r}=0.958778$ and $\omega_{r}=1.879050$ are superimposed upon the nonoscillatory steady mode $\left(\omega_{r}=0\right)$ and are shown in Fig. 4, which presents the correspondence of the frequencies of the damped linear twodimensional eigenmodes of the converged steady-states, with those obtained from discrete Fourier transforms of the DNS.

As it was mentioned before, the first POD topo-mode is able to capture the spatial structure of the leading linear global eigenmode, due to the existence of a strongly dominant linear instability. However, the first POD chrono-mode [see Eq. (12)] presents a single oscillatory frequency $\left(\omega_{r}=0.000976\right)$. Figure 5 presents the

Table 2 Damping rate of the leading stationary eigenmode obtained by solution of the global instability eigenvalue problem and DMD analysis of transient DNS data

\begin{tabular}{|c|c|c|c|c|}
\hline \multicolumn{5}{|c|}{ Mode I } \\
\hline & EVP (4) & DMD (15) & TS (4) & RA (5) \\
\hline$\overline{\Re\{\omega\}}$ & 0 & 0 & 0 & 0 \\
\hline$\widetilde{J}\{\omega\}$ & -0.031714 & -0.031712 & -0.031754 & -0.031703 \\
\hline \multicolumn{5}{|c|}{ Mode II } \\
\hline & $\operatorname{EVP}(4)$ & DMD (15) & $\operatorname{TS}(4)$ & RA (5) \\
\hline$\Re\{\omega\}$ & 0.966158 & 0.963098 & 0.961099 & 0.958778 \\
\hline$\tilde{J}\{\omega\}$ & -0.066754 & -0.064574 & -0.067017 & -0.059983 \\
\hline
\end{tabular}

frequency diagram of this first POD chrono-mode, which contains more than $99.9 \%$ of the kinetic energy. As usual in POD analysis, POD modes travel in pairs. In this problem, energy level and magnitude of second and third topo- and chrono-modes are of the same order. Because of its small amount of energy, they can be considered as small variations of the dominant mode that compose the original flow, and they are not considered relevant case of this study. In summary, it is shown that, in this flow configuration, which is dominated by a few stationary and traveling linearly damped eigenmodes, the frequency and damping characteristics of the leading linear flow perturbations are captured correctly by any of DMD, TS, and RA analysis. Because of the fact that the leading POD eigenmode contains practically all the disturbance energy at the conditions examined, POD analysis can also capture exactly this dominant flow perturbation, such that any of the aforementioned methods may be used if analysis is to focus exclusively on that flow eigenmode.

\section{Instability Analysis Using Stable Very-High-Order Finite-Differences (FD- $q$ )}

Turning to the issue of efficiency of present-day computations of global instability results, the rapid increase of computing hardware capabilities over the last decades may be perceived as having reduced the need to devise time-stepping techniques, because matrix formation and inversion became increasingly less expensive as hardware has improved. Nevertheless, matrix-forming global instability analysis algorithms involving direct dense matrix operations, which require $\mathcal{O}\left(N^{2}\right)$ memory for the matrix storage and $\mathcal{O}\left(N^{3}\right)$ CPU time for the matrix inversion associated with seeking interior eigenvalues, 


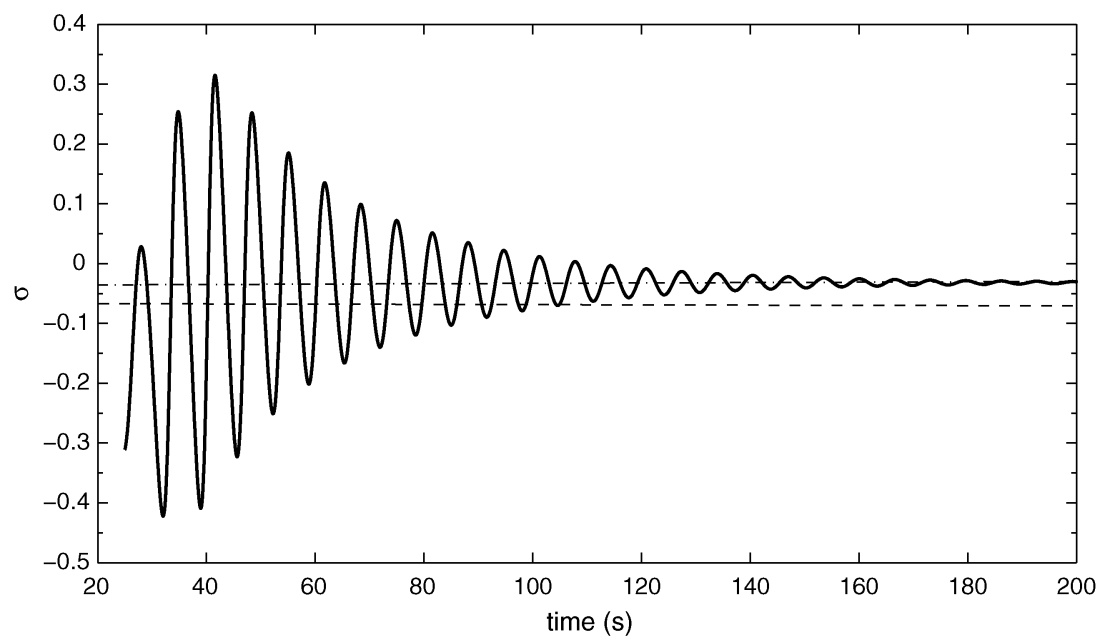

Fig. 3 Dependence of damping ratio $\sigma$ with time showing the exponential decay of two travelling modes $\left(\omega_{r}=0.958778, \sigma=0.059983\right)$ and $\left(\omega_{r}=1.879050, \sigma=0.065227\right)$, superimposed upon the steady mode $\left(\omega_{r} \sim 0, \sigma=0.031703\right)$ at $R e=2000$.

can quickly become very expensive for biglobal hydrodynamic instability problems at moderate Reynolds numbers and are impractical for degrees of freedom, $N$, typical of well-resolved hydrodynamic [7] and especially aeroacoustic [31] global eigenmodes. Crouch et al., in their groundbreaking work on analysis of transonic turbulent flow over an airfoil $[13,14]$, demonstrated that use of parallelizable sparse direct solvers, in conjunction with matrix formation and inversion, is a viable alternative to a time-stepping approach. Gennaro et al. [67] found the effectiveness of sparse direct solvers for biglobal instability problems to depend strongly on whether incompressible or compressible analysis is performed and whether spectral collocation or high-order finite-differences are used for the spatial discretization.
Motivated by these findings, research into high-order finitedifference methods was initiated, posing the question of accuracy before turning attention to efficiency. Besides standard high-order central finite differences, summation by parts [68,69], Padé [70], dispersion-relation preserving [71], and the less-known finitedifference scheme of very-high-order $q$, (FD- $q$ ) of Hermanns and Hernández [58] were pitched against each other, and their results were compared against those delivered by the spectral collocation method based on (standard and coordinate-transformed) Chebyshev-Gauss-Lobatto grid. Figure 6 shows the relative error for the amplification rate of the leading eigenmode of plane Poiseuille flow (PPF) at $R e=10^{4}, \alpha=1$ (Orszag [72]) as obtained by the five methods using double precision arithmetic and the converged

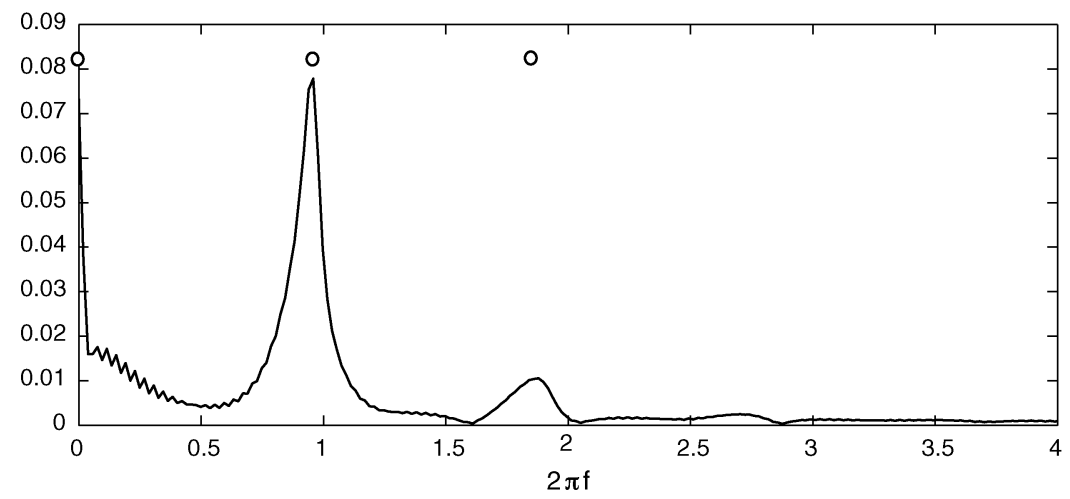

Fig. 4 Correspondence of the frequencies of the damped linear two-dimensional eigenmodes of the converged steady-states obtained from discrete Fourier transforms of the DNS signals at $R e=2000$.

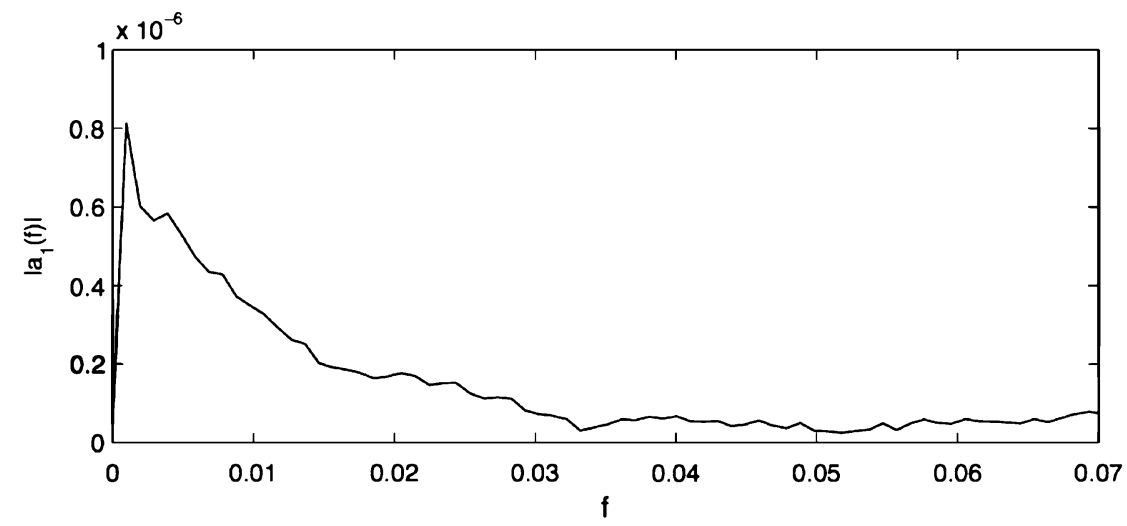

Fig. 5 Frequency diagram of the first POD chrono-mode, Eq. $(12),\left(\omega_{r}=0.000976,0.012695,0.041992\right)$ at $R e=2000$. 


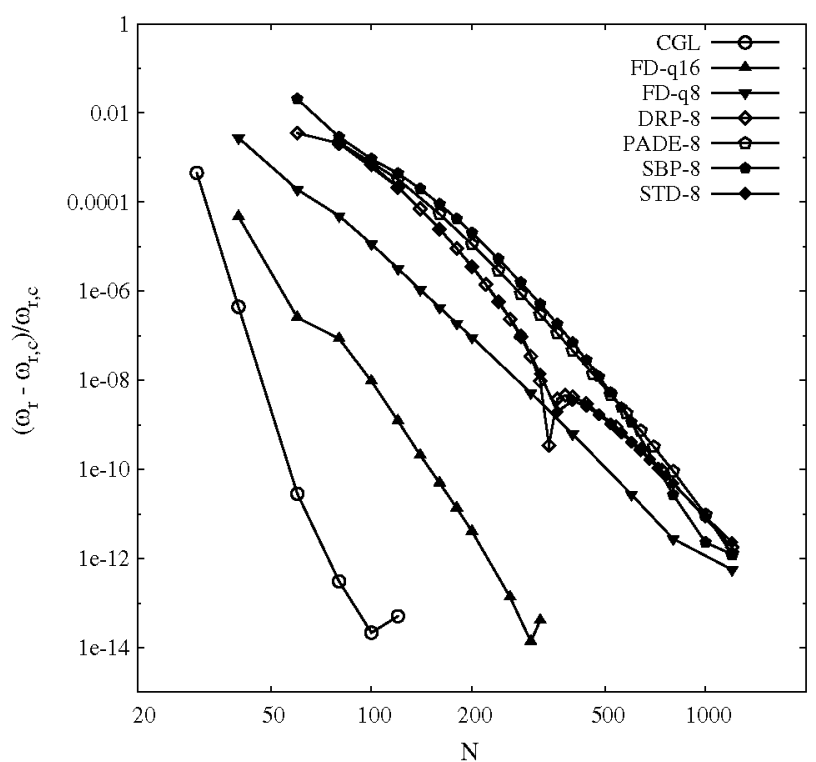

Fig. 6 Relative error for the amplification rate of the leading eigenmode of plane Poiseuille flow at $R e=10^{4}, \alpha=1$ (Orszag [72], Kirchner [73]), obtained by spectral collocation using the ChebyshevGauss-Lobatto grid and high-order finite-difference methods of various orders q: Padé [70], DRP [71], SBP [68,69], and FD-q [58].

result of Kirchner [73], $\omega_{r, c}=0.2375264888204682$ as reference. Figure 7 shows the eigenspectrum of Blasius flow at $R e_{\delta^{*}}=580$, $\alpha=0.179$ (Mack [74]), obtained with spectral collocation based on mapped Chebyshev-Gauss-Lobatto grids, as well as members $q=8$ and $q=16$ of the FD- $q$ family [58]. A brief account of the findings is presented here; further details may be found in Paredes et al. [36].

In the PPF, it is seen that at a given order (here eight, although analogous results have been obtained at all orders) the performance of the better-known high-order FD methods [68-71] is comparable, while the FD- $q$ method is substantially more accurate; to achieve a relative error of $10^{-4}$ in the amplification rate, $N=80$ points are needed by FD- $q 8$ and more than $N=150$ by all other methods. Conversely, and much more importantly from the point of view of the subsequent use of the FD- $q$ methods as the basis for spatial

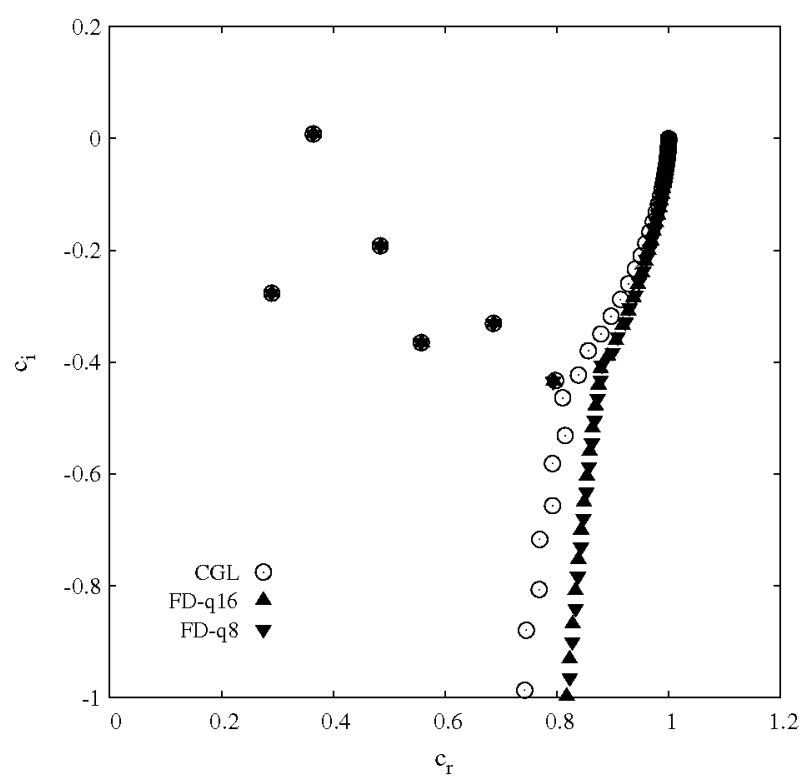

Fig. 7 Eigenspectrum of Blasius flow at $R e_{\delta^{*}}=\mathbf{5 8 0}, \alpha=\mathbf{0 . 1 7 9}$ (Mack [74]), obtained with spectral collocation based on mapped ChebyshevGauss-Lobatto grids, as well as two high-order finite-difference methods [58]. discretization in biglobal, PSE-3D and triglobal instability analyses, at a given affordable level of discretization, say $N=200$ points, Padé, summation by parts (SBP), and dispersion relation preserving (DRP) methods of eighth order produce a relative error of $10^{-5}$ in the recovery of the leading eigenmode, while the error of the FD- $q 8$ method is slightly larger than $10^{-7}$. As the order of the FD- $q$ method is increased, its results approach those of the spectral collocation method, with which the method becomes identical when $q=N$.

At all formal orders examined, in order for the Padé/DRP/SBP schemes to become competitive with FD- $q$, the former need to recover the accuracy lost due to their treatment of the boundary closure if a given bandwidth corresponding to $q$ order is used. Although this is not an issue from the point of view of efficiency when local instability is analyzed, where full eigenspectrum computations are performed using the $Q Z$ algorithm, FD- $q$ has a competitive advantage in performing global instability analysis, where use of sparse matrix libraries is essential and one seeks to use the method having optimal convergence properties between all available having the same nominal order of accuracy and associated sparsity pattern.

The accuracy of the FD-q method is preserved in open flows, as shown in Figs. 6 and 7, where the leading unstable eigenmode and the least stable part of the Blasius eigenspectrum are shown, recovered by the spectral collocation method and $N=129$ Chebyshev-GaussLobatto points, as well as FD- $q 8$ and FD- $q 16$. Even for such relatively low values of $q$, the entire discrete eigenspectrum is seen to be recovered as reliably as by the spectral collocation method, while none of the three methods is capable of capturing the continuous spectrum correctly; as is known analytically, the latter is a vertical line at $c_{r}=1$. Interestingly, even at $q=8$, the discrete approximation of the continuous spectrum is more vertical than that delivered by the collocation method, although, as $q$ increases, the FD- $q$ and spectral results come closer and collapse onto each other at $q=N$.

\section{Three-Dimensional Parabolized Stability Equation Using FD- $q$}

Paredes et al. [36] discuss a step-by-step construction of the analysis tools, validating extensively each of the analysis steps. A parabolized variation of the three-dimensional stability equations (PSE-3D) can be derived if the basic flow can be assumed to experience slow variations along one of the three spatial directions (see Table 1). In this manner, the three-dimensional eigenvalue problem is replaced by an initial value problem that is solved using a marching procedure along the slow direction. The PSE equations can be written in a compact form as

$$
\mathcal{L} \hat{\mathbf{q}}+\mathcal{M} \frac{\partial \hat{\mathbf{q}}}{\partial x}=0
$$

where operators $\mathcal{L}$ and $\mathcal{M}$ act only in $y$ and $z$ but depend on the complex value $\alpha(x)=\alpha_{r}(x)+i \alpha_{i}(x)$, the real part of which is related with the periodicity length along the homogeneous spatial direction $x$ through $\alpha_{r}=2 \pi / L_{x}$, and the imaginary part is the spatial amplification/damping rate. An ambiguity exists in the PSE formulation, in which the changes in amplitude along the slow spatial direction can be contained both in the amplitude function $\hat{\mathbf{q}}$ or in the phase function of the Ansatz (see Table 1). A normalization condition is required to close the formulation of the problem (see Herbert [26] for a review in conventional PSE). In this work, the following normalization condition is used:

$$
\int_{\Omega} \hat{\mathbf{u}}^{\dagger} \frac{\partial \hat{\mathbf{u}}}{\partial x} \mathrm{~d} \Omega=\int_{\Omega} \frac{1}{2} \frac{\partial}{\partial x}|\hat{\mathbf{u}}|^{2} \mathrm{~d} \Omega=0
$$

where $\hat{\mathbf{u}}=(\hat{u}, \hat{v}, \hat{w})^{T}$. This normalization imposes that the kinetic energy of the shape functions remains independent of $x$. Thus, the amplitude growth is absorbed into the phase function.

After developing and validating a standard PSE approach using the FD- $q$ spatial differentiation in both planar and axisymmetric geometries, the spatial biglobal EVP is solved by a companion matrix; the novelty of these results makes them interesting in their 


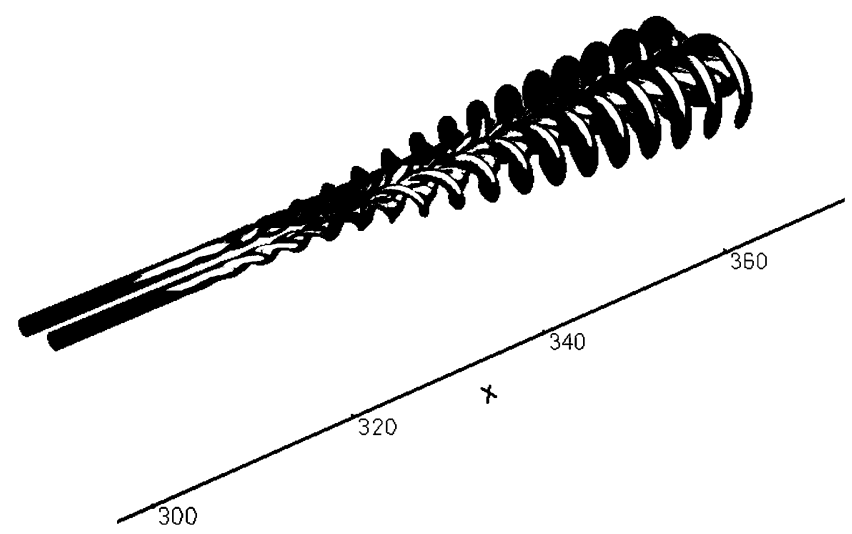

Fig. 8 Isosurface of the flowfield formed by an axially inhomogeneous system of steady laminar vortices and their leading global eigenmode obtained by the linear PSE-3D methodology at $R e=1200$.

own right, although in the work of Paredes et al. [36] they have been used to provide the necessary initialization for the PSE-3D approach. Both the axisymmetric PSE and the PSE-3D codes were validated using the nonparallel model Batchelor vortex. Subsequently, a basic state composed of an axially inhomogeneous pair of counter-rotating (true, axially inhomogeneous) Batchelor vortices has been analyzed using the PSE-3D methodology. The PSE-3D marching integration is initialized by the leading spatial mode of two model Batchelor vortices exposed in [36] with axial velocity defect $\gamma=0.9$, vortex core radius $\delta=1.0$, and swirl parameter $\kappa=-1.14$ at $R e=1200$ and the fixed frequency $\omega=2$. The resulting sought complex eigenvalue of the spatial biglobal EVP is $\alpha_{0}=1.79368-\mathrm{i} 0.589156$. Figure 8 shows the isosurface of the axial velocity flowfield, $\mathbf{q}=$ $\overline{\mathbf{q}}+\varepsilon \hat{\mathbf{q}}$ with $\varepsilon=10^{-4} /\left|u_{0}\right|$, for the leading linear flow perturbation, while more details are provided in the paper of Paredes et al. [36].

\section{E. Triglobal Instability Analysis on a Desktop Computer Using FD- $q$}

Finally, the FD- $q$ method [58] has also been the enabling technology permitting the solution of the triglobal EVP by matrixforming techniques, in which no assumptions of flow homogeneity in

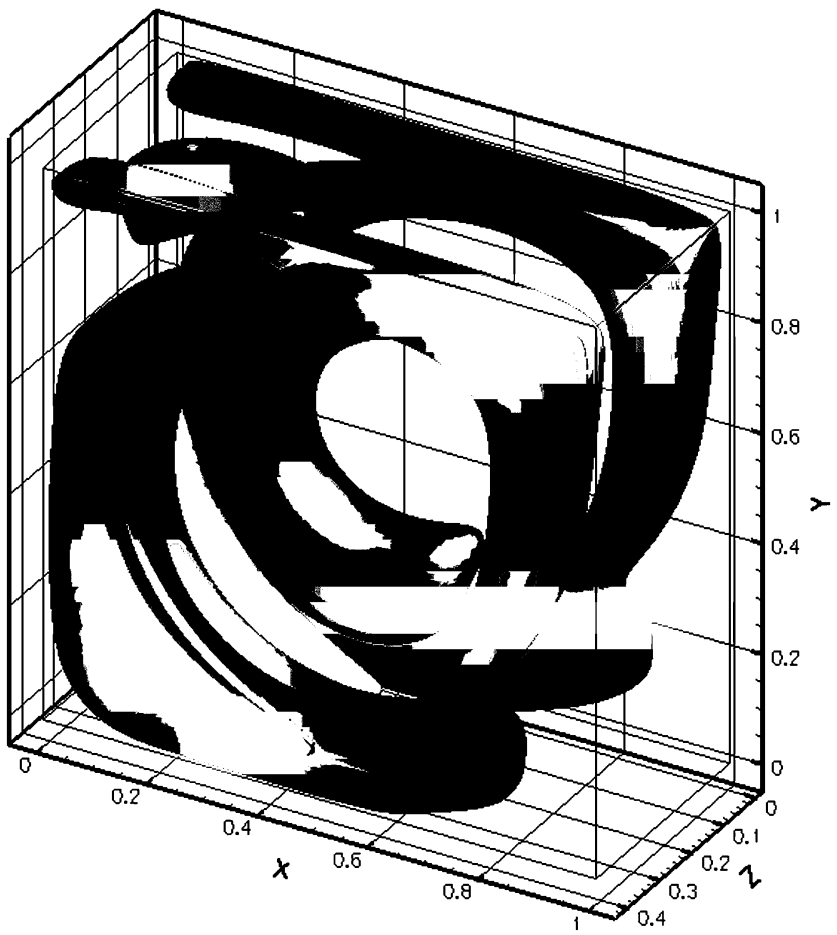

Fig. 9 Leading biglobal eigenmode of lid-driven cavity flow [65] recovered by solution of the triglobal EVP, without assuming spanwise flow homogeneity. Isosurfaces of $\hat{u}$ at $(-0.4,-0.03,0.4)$. any spatial direction are made. Validation was provided by the wellknown leading two-dimensional biglobal eigenmode of lid-driven cavity flow $[65,75,76]$, which was recovered by the triglobal analysis, the latter performed without exploitation of the spanwise invariance of the basic state. A result is shown in Fig. 9, while details are discussed by Paredes et al. [36].

Interestingly, from a computational efficiency point of view, the resolution used for the result shown in Fig. 9 can be compared against that used for the solution of the biglobal EVP $[48,49]$ in both cases forming and storing the respective matrices. The leading dimension in the former EVP has been $4 \times 56^{2} \times 12$, which is of the same order of magnitude as the leading dimension resulting from a typically used resolution in the latter EVP, e.g., $4 \times 340 \times 90$ used to ensure convergence in [49]. The sparse treatment of the matrix discretizing the triglobal EVP, as opposed to the dense parallel approach used for the solution of the biglobal problem meant that a typical dualprocessor desktop with $8 \mathrm{~GB}$ of RAM and $3 \mathrm{MB}$ of L2 cache could be used for the first, in the place of a distributed memory supercomputer with several hundred processors used in our earlier biglobal analysis work $[49,77]$.

\section{Conclusions}

Global linear instability theory has yielded ground-breaking advancements in the understanding of fluid flow instability over or through complex geometries, both in the original modal context and in the more recently incorporated into global instability analysis nonmodal theory. Simplifying assumptions made in the past in order to render instability analysis of a realistic configuration amenable to the classic linear instability theory may now be questioned in the light of the new methodology. If the assumptions of local and quasi-local theories are verified in a context of a global analysis, the former methodologies are bound to prevail as the methods of choice for the analysis on account of their efficiency, otherwise they may now be abandoned in favor of the more complete global instability analysis approach.

The last decade saw significant achievements of the theory, such as the overall first TriGlobal modal analysis, and the first such analysis using matrix-forming techniques, as well as the first global instability analyses of turbulent flow over airfoil geometries. However, most flows in three-dimensional domains with two inhomogeneous spatial directions, and all but a handful of flows in three inhomogeneous spatial directions are yet to be analyzed from a global instability theory point of view, even in the laminar regime. Consequently, the pages of high-impact factor journals are duly filling up at an accelerating pace with results obtained using state-of-the-art computational technologies to address the still formidable numerical tasks associated with the theory. However, contrary to the situation in a flat-plate boundary layer or a shear-layer flow, in both of which the underlying experimentally observed instability modes have been extensively studied in parallel with theoretical efforts for their explanation, what is largely missing from reported analyses of global flow instability is a culture of using experimental reality both as a sanity check of theory and as guidance for its further development. Besides this major concern, other reasons to contain the euphoria surrounding global instability are associated with a list of challenges, some of which discussed explicitly and others alluded to herein, are summarized next.

In contrast to closed systems, theoretically-founded inflow/ outflow boundary conditions for open systems, equally relevant to BiGlobal and TriGlobal instability analysis, are presently missing. To-date only heuristic arguments can be found in the literature regarding the degree by which the various approximations to a theoretically unknown boundary closure affect either the shape of the eigenspectrum and amplitude functions, or the maximal amplification of optimal perturbations, when a modal or a non-modal instability analysis is performed, respectively. A prime case in this point is the Blasius boundary layer, whose eigenspectrum has been exhaustively studied by local and non-local analysis, but is yet to be reconciled with results of global linear theory, other than under conditions mimicking classic linear stability theory approximations. 
Vortical flows, modeled by isolated or systems of (viscous or inviscid) infinitely long axisymmetric tubes of vorticity, is another example of prototype flow the instability of which is well-understood from a classic linear theory point of view. However, it has been shown that using more realistic spatially-diffusing vortices and relaxing the axial homogeneity assumption, instability results are obtained, which are not only quantitatively but also qualitatively different to those delivered by the classic analysis. A new theoretical concept capable of handling basic flow inhomogeneity along the axial direction, in conjunction with strong dependence of the basic state on the plane normal to the vortex axis was introduced in the past and is further discussed in references herein.

Instability analyses of turbulent flows with two homogeneous spatial directions have long been performed in case the dynamics are driven by a single frequency, while analogous linear stability theory in flows in which a broadband turbulence spectrum exists has only recently begun. Despite the obvious qualitative analogies of the proper orthogonal decomposition and global linear theory eigenfunctions in those flows on which both analyses have been attempted so far, no firm theory currently exists to relate the two, although recent evidence suggests that they may be related, as anticipated by the early proper orthogonal decomposition developers. The construction of reduced order models, first for efficient description and then for control of flow at conditions amenable to application of global theory is still at its infancy. Combination of results of flow topology and those delivered by global linear instability analysis constitutes a very powerful tool for quantification of the identified structures and has only been used on two related flows so far; this line of work should be intensified in future.

Contrasting the presently feasible state of affairs against the ultimately desirable targets in global linear instability and motivated by some of the questions posed above, recent work aiming at orderof-magnitude improvements in the numerical solution of the eigenvalue (and singular value) problems governing global flow instability has been introduced. The efficiency gains offered by the stable very high-order finite-differencing scheme used to discretize the related spatial operators has been put to use to obtain accurate PSE-3D and TriGlobal Eigenvalue problem solutions on present-day desktop computers.

\section{Acknowledgments}

Support has been provided by the Spanish Ministry of Science and Innovation through grant MICINN-TRA2009-13648 ("Metodologías computacionales para la predicción de inestabilidades globales hidrodinámicas y aeroacústicas de flujos complejos").

\section{References}

[1] Theofilis, V., "Global Linear Instability," Annual Review of Fluid Mechanics, Vol. 43, Jan. 2011, pp. 319-352. doi:10.1146/annurev-fluid-122109-160705

[2] Theofilis, V., "Advances in Global Linear Instability of Nonparallel and Three-Dimensional Flows," Progress in Aerospace Sciences, Vol. 39, No. 4, 2003, pp. 249-315. doi:10.1016/S0376-0421(02)00030-1

[3] Pierrehumbert, R. T., and Widnall, S. E., "The Two- and ThreeDimensional Instabilities of a Spatially Periodic Shear Layer," Journal of Fluid Mechanics, Vol. 114, Jan. 1982, pp. 59-82. doi:10.1017/S0022112082000044

[4] Pierrehumbert, R. T., "Universal Shortwave Instability of TwoDimensional Eddies in an Inviscid Fluid," Physical Review Letters, Vol. 57, No. 17, 1986, pp. 2157-2159. doi:10.1103/PhysRevLett.57.2157

[5] Tezuka, A., and Suzuki, K., "Three-Dimensional Global Linear Stability Analysis of Flow Around a Spheroid," AIAA Journal, Vol. 44, No. 8, 2006, pp. 1697-1708. doi:10.2514/1.16632

[6] Morzyński, M., and Thiele, F., "Finite Element Method for Global Stability Analysis of 3D Flows," 38th AIAA Fluid Dynamics Conf. and Exhibition, Seattle, WA, AIAA Paper 2008-3865, June 2008.

[7] Bagheri, S., Schlatter, P., Schmid, P. J., and Henningson, D. S., "Global Stability of a Jet in Crossflow," Journal of Fluid Mechanics, Vol. 624, April 2009, pp. 33-44. doi: $10.1017 / \mathrm{S} 0022112009006053$

[8] Giannetti, F., Luchini, P., and Marino, L., "Linear Stability Analysis of Three-Dimensional Lid-Driven Cavity Flow," Proceedings of the 19th Congress of the Italian Association of Theoretical and Applied Mechanics, Aras Edizioni, Ancona, Italy, 14-17 Sept. 2009, pp. 738.1738.10 .

[9] Feldman, Y., and Gelfgat, A., "Oscillatory Instability in a 3D LidDriven Flow in a Cube," Physics of Fluids, Vol. 22, No. 9, 2010, Paper 093602.

doi: $10.1063 / 1.3487476$

[10] Drazin, P. G., and Reid, W. H., Hydrodynamic Stability, Cambridge Univ. Press, New York, 1981.

[11] Schmid, P., and Henningson, D. S., Stability and Transition in Shear Flows, Springer, New York, 2001.

[12] Gaster, M., Kit, E., and Wygnanski, I., "Large-Scale Structures in a Forced Turbulent Mixing Layer," Joumal of FluidMechanics, Vol. 150, Jan. 1985, pp. 23-39. doi: $10.1017 / \mathrm{S} 0022112085000027$

[13] Crouch, J. D., Garbaruk, A., and Magidov, D., "Predicting the Onset of Flow Unsteadiness Based on Global Instability," Journal of Computational Physics, Vol. 224, No. 2, 2007, pp. 924-940. doi:10.1016/j.jcp.2006.10.035

[14] Crouch, J. D., Garbaruk, A., Magidov, D., and Travin, A., "Origin of Transonic Buffet on Aerofoils," Joumal of Fluid Mechanics, Vol. 628, 2009, pp. 357-369. doi: $10.1017 / \mathrm{S} 0022112009006673$

[15] Kitsios, V., Cordier, L., Bonnet, J.-P., Ooi, A., and Soria, J., "On the Coherent Structures and Stability Properties of a Leading-Edge Separated Aerofoil with Turbulent Recirculation," Journal of Fluid Mechanics, Vol. 683, 2011, pp. 395-416. doi:10.1017/jfm.2011.285

[16] Noack, B. R., Afanasiev, K., Morzynski, M., Tadmor, G., and Thiele, F., "A Hierarchy of Low-Dimensional Models for the Transient and PostTransient Cylinder Wake," Joumal of Fluid Mechanics, Vol. 497, 2003, pp. 335-363. doi: $10.1017 /$ S0022112003006694

[17] Rowley, C. W., Mezić, I., Bagheri, S., Schlatter, P., and Henningson, D. S., "Spectral Analysis of Nonlinear Flows," Journal of Fluid Mechanics, Vol. 641, 2009, pp. 115-127. doi:10.1017/S0022112009992059

[18] Ma, Z., Ahuja, S., and Rowley, C. W., "Reduced-Order Models for Control of Fluids Using the Eigensystem Realization Algorithm," Theoretical and Computational Fluid Dynamics, Vol. 25, Nos. 1-4, 2010, pp. 233-247. doi:10.1007/s00162-010-0184-8

[19] Schmid, P. J., "Dynamic Mode Decomposition of Numerical and Experimental Data," Journal of Fluid Mechanics, Vol. 656, 2010, pp. 5-28. doi: $10.1017 /$ S0022112010001217

[20] Noack, B., Tadmor, G., and Morzynski, M., "Low-Dimensional Models for Feedback Flow Control. Part 1: Empirical Galerkin Models," 2nd AIAA Flow Control Conference, Portland OR, AIAA Paper 2004-2408, 28 June-1 July 2004.

[21] Luchtenburg, D. M., Günther, B., Noack, B., King, R., and Tadmor, G., "A Generalized Mean-Fieldmodel of the Natural and High-Frequency Actuated Flow Around a High-Lift Configuration," Journal of Fluid Mechanics, Vol. 623, 2009, pp. 283-316. doi: $10.1017 / \mathrm{S} 0022112008004965$

[22] Tadmor, G., Lehmann, O., Noack, B. R., Cordier, L., Delville, J., Bonnet, J.-P., et al., "Reduced-Order Models for Closed-Loop Wake Control," Philosophical Transactions of the Royal Society. Series A, Mathematical, Physical, and Engineering Sciences, Vol. 369, No. 1940, 2011, pp. 1513-1524.

[23] Bewley, T. R., "Flow Control: New Challenges for a New Renaissance," Progress in Aerospace Sciences, Vol. 37, No. 1, 2001, pp. 21-58. doi:10.1016/S0376-0421(00)00016-6

[24] Kim, J., and Bewley, T. R., "A Linear Systems Approach to Flow Control," Annual Review of Fluid Mechanics, Vol. 39, Jan. 2007, pp. 383-417. doi:10.1146/annurev.fluid.39.050905.110153

[25] Noack, B. R., Morzynski, M., and Tadmor, G., Reduced-Order Modelling for Flow Control, Springer, 2011.

[26] Herbert, T., "Parabolized Stability Equations," Annual Review of Fluid Mechanics, Vol. 29, Jan. 1997, pp. 245-283. doi:10.1146/annurev.fluid.29.1.245

[27] Bertolotti, F. P., Herbert, T., and Spalart, P. R., "Linear and Nonlinear Stability of the Blasius Boundary Layer," Joumal of Fluid Mechanics, Vol. 242, 1992, pp. 441-474. doi: $10.1017 / \mathrm{S} 0022112092002453$ 
[28] Hein, S., Stolte, A., and Dallmann, U. C., "Identification and Analysis of Nonlinear Transition Scenarios Using NOLOT/PSE," Zeitschrift fir Angewandte Mathematik und Mechanik, Vol. 79, No. S1, 1999, pp. 109-112. doi:10.1002/zamm.19990791329

[29] Keller, H. B., "Numerical Solution of Bifurcation and Nonlinear Eigenvalue Problems," Applications of Bifurcation Theory, edited by $\mathrm{P}$. Rabinowitz, Academic Press, 1977, pp. 359-384.

[30] Åkervik, E., Brandt, L., Henningson, D. S., Hœpffner, J., Marxen, O., and Schlatter, P., "Steady Solutions of the Navier-Stokes Equations by Selective Frequency Damping," Physics of Fluids, Vol. 18, No. 6, 2006, Paper 068102 doi: $10.1063 / 1.2211705$

[31] Nichols, J. W., and Lele, S. K., "Global Modes and Transient Response of a Cold Supersonic Jet," Journal of Fluid Mechanics, Vol. 669, 2011, pp. 225-241. doi: $10.1017 / \mathrm{S} 0022112010005380$

[32] Morse, P. M., and Feshbach, H., Methods of Theoretical Physics, Parts 1 and 2, McGraw-Hill, New York, 1953.

[33] Bridges, T. J., and Morris, P. J., "Differential Eigenvalue Problems in Which the Parameter Appears Nonlinearly," Joumal of Computational Physics, Vol. 55, No. 3, 1984, pp. 437-460. doi:10.1016/0021-9991(84)90032-9

[34] Theofilis. V., "Spatial Stability of Incompressible Attachment Line Flow," Theoretical and Computational Fluid Dynamics, Vol. 7, No. 3, 1995, pp. 159-171. doi:10.1007/BF00312360

[35] Rodríguez, D., Tumin, A., and Theofilis, V., "Towards the Foundation of a Global Modes Concept," 6th AIAA Theoretical Fluid Mechanics Conference, Honolulu, AIAA Paper 2011-3603, June 2011.

[36] Paredes, P., Hermanns, M., Le Clainche, S., and Theofilis, V., "Order 10000 Speedup in Global Linear Instability Analysis Using Matrix Formation," Computer Methods in Applied Mechanics and Engineering, accepted for publication. doi:10.1016/j.cma.2012.09.014

[37] Theofilis, V., "On Steady-State Flow Solutions and Their Nonparallel Global Linear Instability," Advances in Turbulence, edited by C. Dopazo, Vol. 8, CIMNE, 2000, pp. 35-38.

[38] Holmes, P., Lumley, J., and Berkooz, G., Turbulence, Coherent Structures, Dynamical Systems and Symmetry, Cambridge Univ. Press, New York, 1996.

[39] Sirovich, L., "Turbulence and the Dynamics of Coherent Structures. Part 1: Coherent Structures," Quarterly of Applied Mathematics, Vol. 45, No. 3, 1987, pp. 561-571.

[40] Sirovich, L., and Kirby, M., "Low-Dimensional Procedure for the Characterization of Human Faces," Journal of the Optical Society of America. A, Optics, Image Science, and Vision, Vol. 4, No. 3, 1987, pp. 519-524. doi:10.1364/JOSAA.4.000519

[41] Sirovich, L., Kirby, M., and Winter, M., "An Eigenfunction Approach to Large Scale Transitional Structures in Jet Flow," Physics of Fluids A, Vol. 2, No. 2, 1990, pp. 127-137. doi: $10.1063 / 1.857815$

[42] Rempfer, D., and Fasel, H. F., "Evolution of Three-Dimensional Coherent Structures in a Flat-Plate Boundary Layer," Joumal of Fluid Mechanics, Vol. 260, 1994, pp. 351-357. doi:10.1017/S0022112094003551

[43] Aubry, N., Guyonnet, R., and Lima, R., "Spatiotemporal Analysis of Complex Signals: Theory and Applications," Journal of Statistical Physics, Vol. 64, Nos. 3-4, 1991, pp. 683-739. doi:10.1007/BF01048312

[44] Koopman, B. O., "Hamiltonian Systems and Transformations in Hilbert Space," Proceedings of the National Academy of Sciences, Vol. 17, No. 5,1931 , pp. 315-318.

[45] Schmid, P. J., "Dynamic Mode Decomposition of Numerical and Experimental Data," Journal of Fluid Mechanics, Vol. 656, 2010, pp. 5-28. doi: $10.1017 / \mathrm{S} 0022112010001217$

[46] Mezić, I., "Spectral Properties of Dynamical Systems, Model Reduction and Decompositions," Nonlinear Dynamics, Vol. 41, Nos. 1-3, 2005, pp. 309-325. doi:10.1007/s11071-005-2824-x

[47] Schmid, P. J., Li, L., Juniper, M., and Pust, O., "Applications of the Dynamic Mode Decomposition," Theoretical and Computational Fluid Dynamics, Vol. 25, 2011, pp. 249-259.

[48] Rodríguez, D., and Theofilis, V., "On the Birth of Stall Cells on Airfoils," Theoretical and Computational Fluid Dynamics, Vol. 25, Nos. 1-4, 2011, pp. 105-118. doi:10.1007/s00162-010-0193-7
[49] Rodríguez, D., and Theofilis, V., "Structural Changes of Laminar Separation Bubbles Induced by Global Linear Instability," Joumal of Fluid Mechanics, Vol. 655, 2010, pp. 280-305. doi: $10.1017 / \mathrm{S} 0022112010000856$

[50] Rowley, C. W., Colonius, T., and Murray, R. M., "POD Based Models of Self-Sustained Oscillations in the Flow Past an Open Cavity," 6th AIAA CEAS Aeroacoustics Conference, Lahaina, HI, AIAA Paper 20001969, June 2000.

[51] Rowley, C. W., Colonius, T., and Murray, R. M., "Model Reduction for Compressible Flows Using POD and Galerkin Projection," Physica. D, Nonlinear Phenomena, Vol. 189, Nos. 1-2, 2004, pp. 115-129. doi:10.1016/j.physd.2003.03.001

[52] Sengupta, T. K., Singh, N., and Suman, V., "Dynamical System Approach to Instability of Flow Past a Circular Cylinder," Joumal of Fluid Mechanics, Vol. 656, 2010, pp. 82-115. doi: $10.1017 / \mathrm{S} 0022112010001035$

[53] Merzari, E., Ninokata, H., Mahmood, A., and Rohde, M., "Proper Orthogonal Decomposition of the Flow in Geometries Containing a Narrow Gap," Theoretical and Computational Fluid Dynamics, Vol. 23, No. 5, 2009, pp. 333-351. doi:10.1007/s00162-009-0152-3

[54] Merzari, E., Wang, S., Ninokata, H., and Theofilis, V., "Biglobal Linear Stability Analysis for the Flow in Eccentric Annular Channels and a Related Geometry," Physics of Fluids, Vol. 20, No. 11, 2008, Paper 114104. doi:10.1063/1.3005864

[55] Oberleithner, K., Sieber, M., Nayeri, C. N., Paschereit, C. O., Petz, C., Hege, H.-C., Noack, B. R., and Wygnanski, I., "Three-Dimensional Coherent Structures in a Swirling Jet Undergoing Vortex Breakdown: Stability Analysis and Empirical Mode Construction," Journal of Fluid Mechanics, Vol. 679, 2011, pp. 383-414. doi: $10.1017 / \mathrm{jm} .2011 .141$

[56] Hussain, A. K. M. F., and Reynolds, W. C., "The Mechanics of an Organized Wave in Turbulent Shear Flow," Journal of Fluid Mechanics, Vol. 41, 1970, pp. 241-258. doi: $10.1017 / \mathrm{S} 0022112070000605$

[57] Theofilis, V., and Le Clainche, S., "Global Linear Instability at the Dawn of its 4th Decade: A List of Challenges (A Practical Guide on How to Contain the Euphoria and Avoid the Oversell)," 6th ALAA Theoretical Fluid Mechanics Conference, Honolulu, AIAA Paper 2011-3291, June 2011.

[58] Hermanns, M., and Hernández, J. A., "Stable High-Order FiniteDifference Methods Based on Non-Uniform Grid Point Distributions," International Journal for Numerical Methods in Fluids, Vol. 56, No. 3, 2008, pp. 233-255.

doi:10.1002/fld.1510

[59] Mack, C. J., Schmid, P. J., and Sesterhenn, J. L., "Global Stability of Swept Flow Around a Parabolic Body: Connecting Attachment-Line and Crossflow Modes," Journal of Fluid Mechanics, Vol. 611, 2008, pp. 205-214.

[60] Gómez, F., Gómez, R., and Theofilis, V., "Coupling Time-Stepping Numerical Methods and Standard Aerodynamics Codes for Instability Analysis of Flows in Complex Geometries," 6th Theoretical Fluid Mechanics Conference, Honolulu, AIAA Paper 2011-3753, June 2011.

[61] Eriksson, L. E., and Rizzi, A., "Computer-Aided Analysis of the Convergence to Steady State of Discrete Approximations to the Euler Equations," Joumal of Computational Physics, Vol. 57, No. 1, 1985, pp. 90-128. doi:10.1016/0021-9991(85)90054-3

[62] Chiba, S., "Global Stability Analysis of Incompressible Viscous Flow," Japan Society of Computational Fluid Dynamics, Vol. 7, No. 1, 1998, pp. $20-48$ (in Japanese)

[63] Mack, C. J., and Schmid, P. J., "A Preconditioned Krylov Technique for Global Hydrodynamic Stability Analysis of Large-Scale Compressible Flows," Journal of Computational Physics, Vol. 229, No. 3, 2010, pp. 541-560. doi:10.1016/j.jcp.2009.09.019

[64] Knoll, D. A., and Keyes, D. E., "Jacobian-Free Newton-Krylov Methods: A Survey of Approaches and Applications," Journal of Computational Physics, Vol. 193, No. 2, 2004, pp. 357-397. doi:10.1016/j.jcp.2003.08.010

[65] Theofilis, V., "Globally Unstable Flows in Open Cavities," 6th AIAA CEAS Aeroacoustics Conference and Exhibit, Lahaina, HI, AIAA Paper 2000-1965, June 2000.

[66] Spalart, P. R., and Rogers, M. M., "Spectral Methods for the NavierStokes Equations with One Infinite and Two Periodic Directions," Journal of Computational Physics, Vol. 96, No. 2, 1991, pp. 297-324. doi:10.1016/0021-9991(91)90238-G

[67] Gennaro, E. M., Rodríguez, D., Medeiros, M. A. F., and Theofilis, V., 
"Linear Instability of Orthogonal Compressible Leading-Edge Boundary Layer Flow," 6th ALAA Theoretical Fluid Mechanics Conference, Honolulu, AIAA Paper 2011-3751, June 2011.

[68] Strand, B., "Summation by Parts for Finite-Difference Approximations for $\mathrm{d} / \mathrm{d} x$," Joumal of Computational Physics, Vol. 110, No. 1, 1994, pp. $47-67$. doi:10.1006/jcph. 1994.1005

[69] Mattson, K., and Nordström, J., "Summation by Parts Operators for Finite Difference Approximations of Second Derivatives," Journal of Computational Physics, Vol. 199, No. 2, 2004, pp. 503-540. doi: $10.1016 /$ j.jcp.2004.03.001

[70] Lele, S. K., "Compact Finite Difference Schemes with Spectral-Like Resolution," Joumal of Computational Physics, Vol. 103, No. 1, 1992, pp. $16-42$. doi:10.1016/0021-9991(92)90324-R

[71] Tam, C. K. W., and Webb, J. C., "Dispersion-Relation-Preserving Finite-Difference Schemes for Computational Acoustics," Joumal of Computational Physics, Vol. 107, No. 2, 1993, pp. 262-281. doi:10.1006/jcph. 1993.1142

[72] Orszag, S., "Accurate Solution of the Orr-Sommerfeld Stability Equation," Journal of Fluid Mechanics, Vol. 50, 1971, pp. 689-703. doi: $10.1017 / \mathrm{S} 0022112071002842$

[73] Kirchner, N. P., "Computational Aspects of the Spectral Galerkin
FEM for the Orr-Sommerfeld Equation," International Journal for Numerical Methods in Fluids, Vol. 32, 2000, pp. 119-137.

[74] Mack, L., "A Numerical Study of the Temporal Eigenvalue Spectrum of the Blasius Boundary Layer," Journal of Fluid Mechanics, Vol. 73, 1976, pp. 497-520. doi:10.1017/S002211207600147X

[75] Albensoeder, S., Kuhlmann, H.C., and Rath, H. J., "Three-Dimensional Centrifugal-Flow Instabilities in the Lid-Driven-Cavity Problem," Physics of Fluids, Vol. 13, No. 1, 2001, pp. 121-136. doi: $10.1063 / 1.1329908$

[76] Theofilis, V., Duck, P. W., and Owen, J., "Viscous Linear Stability Analysis of Rectangular Duct and Cavity Flows," Joumal of Fluid Mechanics, Vol. 505, 2004, pp. 249-286. doi:10.1017/S002211200400850X

[77] Rodríguez, D., and Theofilis, V., "Massively Parallel Numerical Solution of the Biglobal Linear Instability Eigenvalue Problem Using Dense Linear Algebra," AIAA Joumal, Vol. 47, No. 10, 2009, pp. 2449-2459

doi: $10.2514 / 1.42714$ 